\title{
Le livre, les productions audiovisuelles et les mutations culturelles du Golfe à l'Océan
}

Note critique sur Anouk Cohen (2016), Fabriquer le livre au Maroc (Paris, IISMM/Karthala), et Dominique Marchetti (dir.) (2017), avec la collaboration de Julien Paris, La Circulation des productions culturelles : cinémas, informations et séries télévisées dans les mondes arabes et musulmans (Rabat, Centre Jacques-Berque, livre en openedition) Review of Anouk Cohen (2016), Fabriquer le livre au Maroc (Paris, IISMM/ Karthala), et Dominique Marchetti (eds.) (2017), with the collboration of Julien Paris, La Circulation des productions culturelles : cinémas, informations et séries télévisées dans les mondes arabes et musulmans (Rabat, Centre Jacques-Berque, ebook on openedition) Nota critica sobre Anouk Cohen (2016), Fabriquer le livre au Maroc (Paris, IISMM/Karthala), y Dominique Marchetti (dir.) (2017), con la colaboración de Julien Paris, La circulation des productions culturelles : cinémas, informations et séries télévisées dans les mondes arabes et musulmans (Rabat, Centre Jacques-Berque, openedition)

\section{Alain Messaoudi}

\section{(2) OpenEdition}

\section{Journals}

Édition électronique

URL : http://journals.openedition.org/bssg/254

DOI : $10.4000 /$ bssg. 254

ISSN : 2490-9424

Éditeur

Presses universitaires de Vincennes

Référence électronique

Alain Messaoudi, «Le livre, les productions audiovisuelles et les mutations culturelles du Golfe à I'Océan », Biens Symboliques / Symbolic Goods [En ligne], 2 | 2018, mis en ligne le 12 avril 2018, consulté le 04 mars 2021. URL : http://journals.openedition.org/bssg/254 ; DOI : https://doi.org/ $10.4000 /$ bssg.254 


\title{
Le livre, les productions audiovisuelles et les mutations culturelles du Golfe à l'Océan
}

\author{
Alain Messaoudi
}

Le livre d'Anouk Cohen et celui qu'a coordonné Dominique Marchetti diffèrent par leur objet et par leur format : le premier est le fruit d'un travail développé dans le cadre d'une thèse de doctorat portant sur les réalités de la production et des pratiques du livre, à l'échelle du Maroc ; le second rassemble dix-sept auteur.e.s autour de la circulation actuelle des productions culturelles audiovisuelles et cinématographiques, dans le domaine de l'information aussi bien que dans celui de la fiction, au sein d'un espace s'étendant du Maroc à l'Inde.

Les deux ouvrages ont en commun d'analyser des productions culturelles qui connaissent une croissance considérable depuis les années 1990. Cette croissance tient, pour le livre au Maroc, au développement de l'alphabétisation et de la lecture, avec pour effet une augmentation considérable du nombre des publications. Pour les productions audiovisuelles et cinématographiques, elle répond à une demande grandissante, liée à l'importante mutation technologique qui a permis le développement des télévisions satellitaires et d'Internet. L'ouvrage dirigé par Dominique Marchetti s'attache à mieux faire comprendre les modalités de ces transformations, qui se sont inscrites dans un contexte de reconfigurations politiques à la suite de l'effondrement du bloc soviétique. II rappelle que ces trente dernières années ont vu la montée en puissance des pays du Golfe et, dans une moindre mesure, de la Turquie, et le déclin relatif de l'Égypte sur le « marché des biens culturels populaires " (Marchetti $2017: 7$ ). L'ouvrage se concentre sur les productions culturelles qui touchent des publics très larges ou des milieux sociaux « faiblement dotés en capitaux économiques et culturels » (Marchetti 2017 : 9). Dans leur avant-propos, Dominique Marchetti et Julien Paris insistent sur la complexité des enjeux liés aux médias : s'ils sont des espaces de pouvoir, ce n'est pas tant par leur puissance intrinsèque que comme carrefours stratégiques entre différents espaces politiques et économiques eux-mêmes lieux de pouvoir. 
Les deux livres mettent en avant un travail fondé sur l'enquête de terrain. Cette enquête est au cœur de l'ouvrage d'Anouk Cohen, qui se nourrit d'une longue pratique d'observation participante dans les métropoles de Rabat et de Casablanca - le préambule du livre, intitulé "Se laisser porter par le terrain ", rappelle de façon très intéressante le processus et les conditions de la recherche, depuis les débuts de l'enquête en 2004. L'observation empirique des pratiques sociales semble parfois une revendication plutôt qu'un usage courant chez certains des auteurs rassemblés par Dominique Marchetti, ce qui tient peut-être à une forte inscription dans le champ disciplinaire des sciences de l'information et de la communication, malgré la volonté de prendre en considération dans l'analyse les personnes qui contribuent à la structuration d'une « économie culturelle globale » ainsi que la « matérialité » des flux, pour reprendre les formulations de la postface signée par Tristan Mattelart (Marchetti 2017 : 274).

Avant de dégager quelques thèmes partagés par les deux livres, il est nécessaire de les présenter chacun brièvement.

Préfacé par Roger Chartier, qui souligne des rapprochements possibles avec les réalités du livre dans les sociétés d'Ancien Régime (faible spécialisation, caractère familial des entreprises, importance du livre religieux, censure...), malgré le contexte mondial fort différent, l'ouvrage d'Anouk Cohen est solidement construit en trois parties. II adopte une démarche régressive qui reprend le parcours de l'enquête, en partant de la présence du livre dans l'espace public ("Les lieux du livre et ses publics »), avant d'étudier sa mise en forme par les éditeurs-imprimeurs ( $2^{\mathrm{e}}$ partie : «Confectionner un livre ») et sa conception par l'auteur ( $3^{e}$ partie : "Écrire »). Après une introduction qui rappelle la genèse du projet et donne une idée précise de l'historiographie existante, la première partie nous emmène sur les lieux de vente (des étals sur

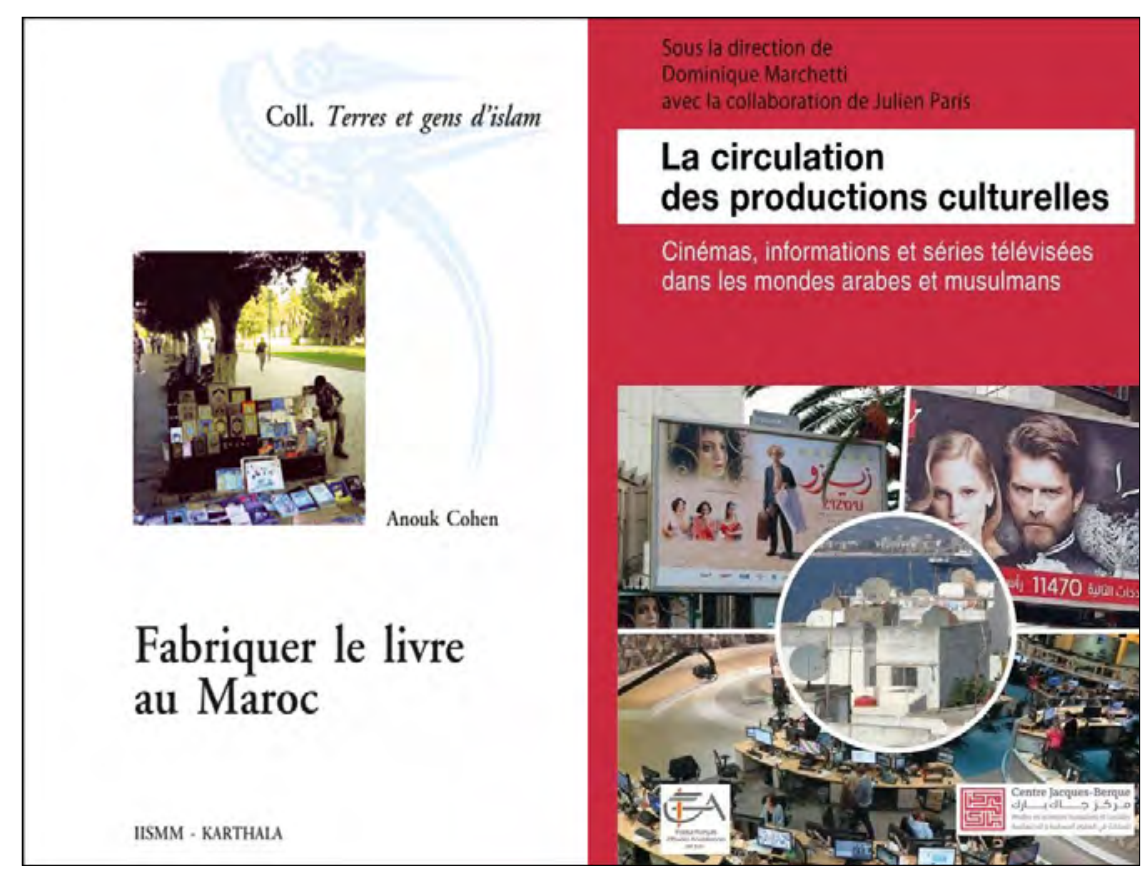

Figure 1. Couverture des ouvrages recensés

À gauche : première de couverture de l'ouvrage d'Anouk Cohen (2016). Fabriquer le livre au Maroc (Paris, IISMM/Karthala).

À droite : première de couverture de l'ouvrage publié sous la direction de Dominique Marchetti (2017). La Circulation des productions culturelles : cinémas, informations et séries télévisées dans les mondes arabes et musulmans (Rabat, Centre Jacques-Berque)

les trottoirs aux librairies, en passant par les kiosques à journaux et les papeteries) [chap. 1] et dans les bibliothèques, qu'elles soient publiques (des institutions peu développées, sinon pour un usage strictement scolaire et utilitaire) ou privées (avec des collections gérées par des fondations qui les ouvrent au public) [chap. 2]. Elle aborde aussi la présence des livres dans les maisons, avec, dans les intérieurs les plus riches, la mise en scène de collections reliées d'ouvrages classiques arabes au sein du « salon marocain » où 
I'on reçoit. Un chapitre est réservé aux formes diverses du Coran, en fonction de ses différents usages : il peut être un signe de prestige ou de piété, l'objet d'un cadeau, ou bien un instrument de lecture sérieuse chez soi (ce qui met au premier plan la lisibilité) ou dans les transports (ce qui suppose sa maniabilité) [chap. 3]. Ce chapitre permet d'introduire une typologie de la lecture et d'identifier des mouvements nouveaux, avec le développement des récits individuels et de la littérature de jeunesse, où l'on peut voir autant de signes de l'affirmation d'une pratique de la lecture comme processus de construction de soi («Lire en ville », chap. 4). Dans la deuxième partie, Anouk Cohen rappelle que le métier d'éditeur n'existe pas au Maroc au sens qu'on donne aujourd'hui à ce mot en Europe. La fabrication et la diffusion du livre en arabe sont concentrées dans "Le souk du livre » (chap. 5) à Rabat, avec des dynasties de libraires, qui entretiennent entre eux des relations de concurrence et de partenariat à la fois, ayant une activité de commanditaires (pour des adaptations généralement « piratées » à destination du marché marocain de livres déjà édités au Machreq) et de diffuseurs. "Être éditeur » (chap. 6) correspond à des réalités fort contrastées, avec un marché du livre en français plus limité et plus concurrentiel que celui du livre en arabe, lui-même polarisé entre un grand nombre de petits éditeurs souvent éphémères et deux entreprises commerciales qui doivent leur importance au livre scolaire. On trouve des profils d'éditeurs militants (souvent d'anciens membres de l'opposition politique au régime) ou intellectuels (avec des auteurs passés à l'édition). «Faire de la politique » (chap. 7) rappelle l'enjeu de l'édition comme soft power pour les autorités politiques, en soulignant l'importance des subventions, publiques ou privées, marocaines ou étrangères, qui conditionnent souvent les publications, et le rôle des foires du livre, qui permettent de rendre visible la production éditoriale en l'absence de diffuseurs. Les deux chapitres suivants, "La mise en pages : entre composition et bricolage » et « L'impression du livre : savoir-faire et techniques ", sont consacrés aux aspects techniques de la fabrication (chap. 8 et 9 ). Pour toucher une large clientèle, le coût de la production doit être faible, au risque d'une qualité médiocre. Plutôt que d'investir dans des équipements coûteux et dans la formation des employés, les éditeurs tendent à sous-traiter le travail spécialisé à l'étranger. La troisième partie du livre, consacrée aux pratiques d'écriture, s'ouvre sur la question de l'impact de la langue - arabe ou français - dans la perception des textes : elle est abordée à partir d'un cas de censure étatique ayant frappé un hebdomadaire publié en arabe dialectal, Nîchân, suite à des protestations exprimées dans les milieux de l'islam politique, quand des propos équivalents avaient pu être publiés dans la presse francophone sans susciter aucune réaction (chap. 10, « La langue du silence »). À travers l'exemple de la publication, dans la presse puis sous forme de livres, de récits carcéraux (chap. 11: "Le livre archive. Le cas des témoignages d'anciens prisonniers politiques ") et celui de la multiplication des récits de vie (chap. 12 : "Écrits intimes »), Anouk Cohen repère les signes d'une affirmation de l'individualité, présentée comme une garantie d'authenticité, qui marquerait un processus d'émancipation par rapport au groupe et à ses normes.

Les quatorze contributions (artificiellement présentées comme autant de chapitres) qui constituent $L a$ Circulation des productions culturelles sont elles aussi organisées en trois parties. La première séquence est centrée sur le journalisme d'information et les effets du développement des circulations transnationales sur ses pratiques. Elle retrace la montée en puissance des chaînes d'information panarabes (dont la chaîne qatarie Al Jazeera) et des médias en ligne, progression retracée dans le premier chapitre dû à Tourya Guaaybess. Elle rappelle aussi que cette montée s'est accompagnée de celle du sous-espace arabophone (avec une domination du Golfe sur le Maghreb) dans l'activité des agences de 
presse occidentales, parvenues à conserver leurs positions dans la production de l'actualité, comme le montre Dominique Marchetti. Dans cette même séquence, Enrico de Angelis analyse les effets de la superposition des nouvelles techniques digitales, smartphones et Internet, aux anciens modes de production et de diffusion de l'information (la production de l'information tendrait à devenir plus locale, via des journalistes « citoyens » ou freelance, tandis que la consommation s'internationaliserait). Olivier Koch s'intéresse quant à lui à la mise en œuvre des projets internationaux de formation visant les journalistes après les « printemps arabes » de 2011, à travers le cas de la Tunisie (l'offre n'a pas tenu compte des besoins réels, mais a permis aux formateurs de se constituer un réseau et aux journalistes locaux d'accumuler un capital symbolique). La deuxième partie rassemble des études portant sur les échanges (importations et exportations) en matière de programmes télévisuels (séries, documentaires) et cinématographiques, en s'attachant à leur dimension politique et diplomatique (soft power). L'exemple de l'exportation des séries turques, qui véhiculent des valeurs familiales et nationales obéissant à une économie morale islamique adaptée au public visé à l'étranger, rappelle l'importance des régulations mises en œuvre à l'échelle nationale, préalablement à la diffusion internationale des produits (Julien Paris). L'investissement d'Al Jazeera dans la production de films documentaires sur des sujets historiques ou de société permet de souligner sa volonté d'affirmer une identité culturelle arabe spécifique, bien que ces productions reprennent formellement des modèles préétablis en Occident (Michel Tabet). On trouvera aussi dans cette partie une analyse de la distribution des films indiens au Moyen-Orient, visant en priorité le public de la diaspora travaillant dans le golfe Arabo-Persique, avec l'implantation en 2004 d'un bureau de la principale compagnie cinématographique indienne à Dubaï, l'émirat trouvant intérêt à accueillir une activité permettant de préparer un futur marqué par la fin de l'exploitation pétrolière
(Némésis Srour). La troisième partie, intitulée « Les marchés médiatiques nationaux dans tous leurs états ", se concentre sur les politiques étatiques en matière de production et de diffusion audiovisuelles et cinématographiques, à travers l'exemple de la production de séries et de films télévisés au Maroc (Catherine Miller), les logiques de la distribution des films dits « maghrébins » en France (Abdelfettah Benchenna) et la signification des nouvelles instances de régulation des chaînes de télévision instaurées au Maroc depuis 2002-2004 et en Tunisie depuis 2011-2012, entre manifestation d'une rupture avec l'ancienne gestion autoritaire du secteur audiovisuel et redéploiement d'une souveraineté de l'État menacée par la montée en puissance des chaînes transnationales (Enrique Klaus).

Les deux livres permettent de façon différente de mieux cerner les conséquences de la multiplication de la production et des circulations transnationales en matière de biens culturels au MoyenOrient. On en retiendra en particulier deux aspects : l'impact de la transnationalisation sur les pouvoirs centraux des États-nations, et plus généralement sur la capacité d'action de ces États ; ses effets sur les usages linguistiques et l'affirmation des identités culturelles. Dans le domaine du livre, mais aussi dans celui des médias et du cinéma, les États ne semblent pas aussi affaiblis qu'on pourrait l'attendre. Anouk Cohen montre qu'au Maroc l'État reste le pivot de l'organisation d'un monde du livre qui se caractérise par sa faible spécialisation professionnelle, sans maisons d'édition au sens complet du terme, et sans diffuseurs ; la situation semble équivalente dans le domaine du cinéma, en matière de maisons de production et de distributeurs. Ce sont les institutions étatiques qui dominent le marché de l'édition scolaire, distribuent les subventions et organisent les foires et salons du livre. Dans le domaine de la production audiovisuelle et cinématographique, la transnationalisation des médias ne s'accompagne pas non plus 
nécessairement d'une déprise des États. Le pouvoir de censure reste une réalité, non tant par l'application d'une censure explicite (présente au Maroc, mais dont la mise en œuvre est semble-t-il avant tout l'effet de pressions extérieures, de la part d'un secteur de l'opinion publique, en l'occurrence les sympathisants d'un islam politique) qu'à travers une action indirecte de la part d'États qui conservent une forte capacité d'action dans la production et la commercialisation. On le voit à travers le cas de la Turquie, où Julien Paris révèle les processus de censure invisible qui sont à l'œuvre, le pouvoir ayant la capacité de contrôler la production des séries télévisées du fait de la concentration qui caractérise le secteur de l'industrie des médias. La méfiance éprouvée par les professionnels turcs du cinéma envers la puissance de l'État serait d'ailleurs, selon Romain Lecler et Jean-François Polo, une des explications de l'échec du projet d'exportation du modèle français du Centre national de la cinématographie (CNC) en Turquie. La capacité d'action des États s'exerce aussi en dehors de leurs frontières. Cela vaut pour la Turquie et l'Inde, exportateurs de séries télévisées, comme pour l'Arabie saoudite et les États du Golfe, qui subventionnent la publication et la distribution de livres en langue arabe au Maroc. Cela vaut aussi pour les États européens, en particulier pour la France au Maghreb. Anouk Cohen rappelle l'importance de son aide au Maroc : le bureau du livre du Service de coopération et d'action culturelle français subventionne plus du tiers des livres d'expression française et des traductions qui y sont publiés chaque année (Cohen 2016 : 223-229). La contribution d'Abdelfettah Benchenna rappelle par ailleurs l'implication française dans la promotion d'un « cinéma maghrébin »: les films, pour la plupart des films d'auteurs dont la coproduction implique la France, voient souvent le jour grâce au dispositif français d'aide à la production, dispositif qui n'a pas de véritable équivalent dans les pays du Maghreb, sinon sous une forme limitée au Maroc. Reste à mieux connaître le public de ces films qui ne dépassent généralement pas les 50 000-100 000 entrées en cas de succès : s'agit-il avant tout de Français entretenant des liens avec le Maghreb du fait de leur origine ou de leur histoire ? Cette question pourrait recouper celle du lectorat de la « littérature maghrébine d'expression française ».

Les deux ouvrages permettent aussi de mesurer l'impact des transformations du monde de l'édition et des médias sur l'affirmation des identités culturelles et sur les usages linguistiques, avec le développement d'une production dans la langue arabe du pays. Nicolas Dot-Pouillard observe la réaffirmation d'un modèle de journalisme politiquement engagé, qui ne prétend pas produire une information neutre, en analysant le discours d'Al Jazeera, fondée fin 1996, et d'Al Mayadeen, deux chaînes de télévision à la fois parentes et politiquement opposées (Al Mayadeen, établie au Liban, a été fondée en 2012 par des journalistes d'Al Jazeera à la sensibilité nationaliste arabe, tiers-mondiste et anti-impérialiste, en rupture avec la ligne éditoriale de la chaîne à propos de la situation en Libye, en Syrie et au Bahreïn). Ce journalisme engagé fait écho à des débats intenses pendant la Guerre froide, autour de la critique de la domination des agences de presse occidentales en matière d'information. On retrouve cette aspiration à affirmer un regard arabe sur le monde dans la production de films documentaires dans laquelle le groupe Al Jezeera s'est investi depuis 2003, bien que ces films restent formellement prisonniers de modèles préétablis en Occident, comme le rappelle la contribution de Michel Tabet. Mais se pose aussi la question de la définition de ce regard: fait-il une place au Maghreb, ou renforce-t-il la domination d'un point de vue spécifique au Golfe, malgré l'intégration croissante de journalistes maghrébins dans les chaînes panarabes?

La problématique de l'identité culturelle est aussi centrale dans le cas de l'édition au Maroc. Anouk Cohen fait le constat d'univers spécifiques, sinon cloisonnés, en fonction de la langue dans 
laquelle sont formulées les productions intellectuelles, textuelles, médiatiques, cinématographiques, malgré quelques recoupements - l'existence de producteurs qui utilisent le canal de plusieurs langues, la présence de traductions et de versions multilingues. Elle montre que le monde du livre à Casablanca et à Rabat n'est pas unifié : les professionnels du livre en français et ceux du livre en arabe évoluent dans des univers distincts, malgré l'existence de quelques éditeurs travaillant dans les deux langues, et d'une minorité de lecteurs dont la pratique se partage entre elles. Le clivage est perceptible à travers les lieux de vente (les librairies arabes sont concentrées dans le quartier des habous à Rabat, un quartier construit à l'époque du Protectorat dans un style marocain, alors que les librairies françaises se dispersent dans des quartiers résidentiels habités par une population aisée), à travers aussi les circuits de commercialisation, les éditeurs, les auteurs et les lecteurs qui sont pour la plupart spécialisés dans une langue. L'édition des livres en arabe ne vise pas la même clientèle que celle des livres en français : les premiers, pour être rentables, doivent trouver un public nombreux (et donc afficher des prix bas), tandis que les livres en français, qui visent une clientèle aux moyens financiers supérieurs, peuvent être vendus en plus petit nombre à un prix plus élevé. La censure elle-même, qu'il s'agisse d'une censure d'État ou d'une censure informelle, imposée par des forces sociales ou politiques, n'opère pas de la même façon pour le livre arabe et pour le livre français. Elle est plus sensible pour les textes publiés en arabe, du fait de la charge symbolique de la langue arabe, y compris dans sa version marocaine dârija. L'emploi de la dârija, qui reste limité dans le monde de l'imprimé, et tout particulièrement du livre, s'est en revanche affirmé dans les productions audiovisuelles. Depuis les années 2000, la chaîne privée $2 \mathrm{M}$ a non seulement fait le choix d'un doublage en dârija pour les séries télévisées importées qu'elle diffuse, mais elle s'est également mise à produire des émissions (fictions, téléréalités) en dârija. En rompant avec la politique étatique de promotion de l'arabe littéral « standard », et en bravant des résistances exprimées au sein du gouvernement par un ministre de la Communication membre du Parti de la justice et du développement, la chaîne a répondu à une demande du public : en témoignent des taux d'audience bien supérieurs à ceux des feuilletons doublés en arabe classique. Catherine Miller, qui analyse le phénomène dans sa contribution à l'ouvrage dirigé par Dominique Marchetti, y voit l'affirmation d'une nouvelle définition de la marocanité. Elle rejoint en cela les conclusions d'Anouk Cohen sur les profondes redéfinitions de la société marocaine dont témoigne la production imprimée, liée désormais au monde du numérique par des liens de concurrence et de complémentarité (les blogs en fournissent un exemple). La signification sociale de la lecture n'est plus tant un acte public qu'une activité relevant de la sphère du privé, une mutation qui s'est accompagnée du développement de nouvelles formes de subjectivité, malgré le maintien de conventions littéraires et linguistiques, plus fortes dans le domaine de l'arabe que dans celui du français.

En analysant les formes d'écriture et de lecture au Maroc, et en examinant les mondes de l'édition et de la production médiatique, ces deux livres, chacun à sa façon, permettent de saisir certains aspects des rapides mutations culturelles que connaissent des sociétés du monde arabe, traversées de circulations internationales. Ils sont par certains aspects complémentaires. En croisant les perspectives des historiens du livre et celles des sociologues de l'écriture et de la lecture, Anouk Cohen a fait le choix d'une approche intuitive qui entend rendre le monde du livre intelligible à travers une description méthodique. La qualité de son travail tient à l'acuité de ses observations participantes qui, en s'échelonnant sur une durée longue de plusieurs années, lui ont permis d'acquérir une véritable familiarité avec son objet. L'ouvrage coordonné par Dominique Marchetti a une ambition différente : il ne s'agit pas 
de rendre compte de la façon la plus complète possible d'un monde professionnel et d'une activité sociale, mais de repérer, à travers plusieurs cas exemplaires, des rapports de force et une économie politique s'exerçant par l'intermédiaire des médias. L'enquête au long cours d'Anouk Cohen sur le livre au Maroc invite à une lecture plus continue que l'hétérogénéité relative du bouquet de recherches rassemblé sous la forme d'une publication numérique par Dominique Marchetti. Mais l'une et l'autre apportent une contribution substantielle à un domaine d'études appelé à se développer.

Alain Messaoudi

Université de Nantes - CRHIA (Centre de recherches en histoire internationale et atlantique) / InVisu 